\title{
Quantitative analysis of renal blood flow during thoracic endovascular aortic repair in type B aortic dissection using syngo iFlow
}

\author{
Kun Fang ${ }^{1 \#}$, Jiawei Zhao ${ }^{1 \#}$, Mingyao Luo ${ }^{1 \#}$, Yunfei Xue ${ }^{1}$, Hui Wang ${ }^{2}$, Luming Ye ${ }^{3}$, Xuelan Zhang ${ }^{4}$, \\ Liancun Zheng ${ }^{4}$, Chang Shu ${ }^{1,2} \wedge$
}

${ }^{1}$ State Key Laboratory of Cardiovascular Disease, Center of Vascular Surgery, Fuwai Hospital, National Center for Cardiovascular Diseases, Chinese Academy of Medical Sciences and Peking Union Medical College, Beijing, China; ${ }^{2}$ Department of Vascular Surgery, The Second Xiangya Hospital of Central South University, Changsha, Hunan, China; ${ }^{3}$ Department of Advanced Therapy, Siemens Healthineers, Beijing, China; ${ }^{4}$ School of Mathematics and Physics, University of Science and Technology Beijing, Beijing, China

Contributions: (I) Conception and design: K Fang, J Zhao, M Luo; (II) Administrative support: C Shu; (III) Provision of study materials or patients: Y Xue, H Wang; (IV) Collection and assembly of data: J Zhao, L Ye; (V) Data analysis and interpretation: X Zhang, L Zheng; (VI) Manuscript writing: All authors; (VII) Final approval of manuscript: All authors.

\#These authors contributed equally to this work.

Correspondence to: Chang Shu, MD. Center of Vascular Surgery, Fuwai Hospital, National Center for Cardiovascular Diseases, Chinese Academy of Medical Sciences and Peking Union Medical College, Beijing, China. Email: changshu@vip.126.com.

Background: Currently, the thoracic endovascular aortic repair is the recommended clinical treatment for type B aortic dissections. Unfortunately, malperfusion or ischemia of the kidneys is a major complication of type B aortic dissections. Despite this, few studies have focused on the effects of thoracic endovascular aortic repair on blood flow in renal arteries and parenchyma. This current investigation used novel realtime imaging software to quantitatively analyze the hemodynamic changes in renal artery blood flow and perfusion before and after stent graft placement.

Methods: A total of 51 patients with type B aortic dissection undergoing thoracic endovascular aortic repair between April 2017 and September 2019 were retrospectively recruited. The pre-and post-procedural digital subtraction angiography images were converted into color-coded maps using syngo iFlow for quantitative comparison. Time-intensity curves and related parameters, including the average peak ratio (avg.Pr), average delayed time to peak (avg.dTTP), and average area under the curve ratio (avg.AUCr) of the renal arteries and renal cortex were obtained and analyzed. Wilcoxon signed-rank test was used to compare iFlow parameters before and after endovascular repair. Spearman correlation analyses were performed to study iFlow parameters and renal function parameters and the estimated glomerular filtration rate (eGFR) and blood urea nitrogen (BUN).

Results: A total of 102 images including 51 pre-operative and 51 post-operative image datasets were successfully post-processed. Following endovascular repair, syngo iFlow showed a significant 33.0\% increase in avg. $\operatorname{Pr}(\mathrm{P}<0.001)$ and a significant $35.1 \%$ increase in avg.AUCr $(\mathrm{P}<0.001)$ in the renal artery. Additionally, there was a significant $12.2 \%$ decrease in the avg.dTTP $(\mathrm{P}=0.001)$, a significant $24.5 \%$ increase in avg.Pr $(\mathrm{P}=0.004)$, and a significant $38.3 \%$ increase in avg.AUCr $(\mathrm{P}=0.009)$ in the renal cortex. Spearman correlation analysis showed that after endovascular repair there was a significant correlation between the avg.Pr of the renal artery and eGFR $(r=0.30 ; P=0.0349)$, the avg.Pr of the renal cortex and eGFR $(r=0.30 ; P=0.0300)$, and

\footnotetext{
^ ORCID: 0000-0003-2066-1183.
} 
the avg. AUCr of the renal cortex and BUN (r=0.31; P=0.0289).

Conclusions: syngo iFlow provided a novel quantitative method for evaluating renal hemodynamic changes in patients with type B aortic dissection undergoing endovascular treatment. Time-intensity curve parameters may facilitate the intraprocedural evaluation of renal blood flow and perfusion to complement the color-coded map.

Keywords: Thoracic endovascular aortic repair (TEVAR); type B aortic dissection (TBAD); syngo iFlow; renal blood flow; renal parenchymal perfusion

Submitted Aug 30, 2020. Accepted for publication Apr 09, 2021.

doi: 10.21037/qims-20-992

View this article at: http://dx.doi.org/10.21037/qims-20-992

\section{Introduction}

Malperfusion or ischemia of the kidney is a significant complication of type B aortic dissection (TBAD), which adversely affects patients' prognosis. It is caused by dynamic and/or static flow obstruction of the renal artery due to intimal tear or compressed true lumen of dissection (1). Currently, thoracic endovascular aortic repair (TEVAR) is the recommended treatment for TBAD, especially for complicated TBAD with end-organ ischemia, as TEVAR can seal not only the primary tear but also dilate the compressed true lumen, which improves blood flow in the distal aorta and renal arteries simultaneously $(2,3)$.

There are currently several methods used to measure renal perfusion and blood flow in the renal arteries, such as ultrasound, magnetic resonance imaging (MRI), and enhanced computed tomography (CT), all of which have certain limitations that are difficult to use in clinical practice. At present, renal malperfusion is initially diagnosed using traditional digital subtraction angiography (DSA) combined with pre-operative CT and clinical manifestations. However, this method is not quantitative and mainly relies on the density of contrast judged by visual assessment.

syngo iFlow (Siemens Healthineers, Forchheim, Germany) is a digital color-coded DSA and represents a novel angiography imaging tool for instant intra-operative quantitative analysis. Previous studies have reported the application and practical value of syngo iFlow in evaluating the transcatheter embolization of tumors and interventional therapies for cerebrovascular diseases (4-6).

This study quantitatively analyzed the hemodynamic changes in renal artery blood flow and perfusion before and after stent-graft placement during TEVAR using syngo iFlow in patients with TBAD.

\section{Methods}

\section{Patients}

Between April 2017 and September 2019, a total of 403 patients with TBAD underwent TEVAR surgery in our department. The clinical data, together with the preand post-operative aortic angiography (with eligible and satisfactory image quality) from these patients, were retrospectively collected and analyzed. A total of 352 patients were excluded from this study, as shown in Figure 1. Finally, a total of 51 cases were included in this investigation. This study was conducted following the Declaration of Helsinki (as revised in 2013). The retrospective study was approved by the National Center for Cardiovascular Diseases of China, the Ethics Committee (NO. 2018-1119), and the institutional review board. Patient informed consent was waived due to the retrospective nature of the study.

\section{Treatment procedure and DSA protocol}

All TEVAR treatments followed standard procedures, as previously described (3). DSA was performed under general anesthesia using the same angiographic system for each patient (Artis Zeego; Siemens Healthcare, Forchheim, Germany) with the following DSA settings: $70 \mathrm{kV}$, $3.000 \mu \mathrm{Gy} /$ frame, pulse width $36.8 \mathrm{~ms}$, and 7.5 frames/s. An anteroposterior DSA image (field of view, $48 \mathrm{~cm}$ ), which should be sufficient to cover both kidneys, was obtained before and after graft deployment, with a 5 -Fr pigtail catheter tip positioned in the true lumen at the level of the T8 vertebral body. During each DSA acquisition, $20 \mathrm{~mL}$ of contrast (Visipaque $320 \mathrm{mg} \mathrm{I} / \mathrm{mL}$; GE Healthcare, Cork, Ireland) was injected at a rate of $18 \mathrm{~mL} / \mathrm{s}$ using a 


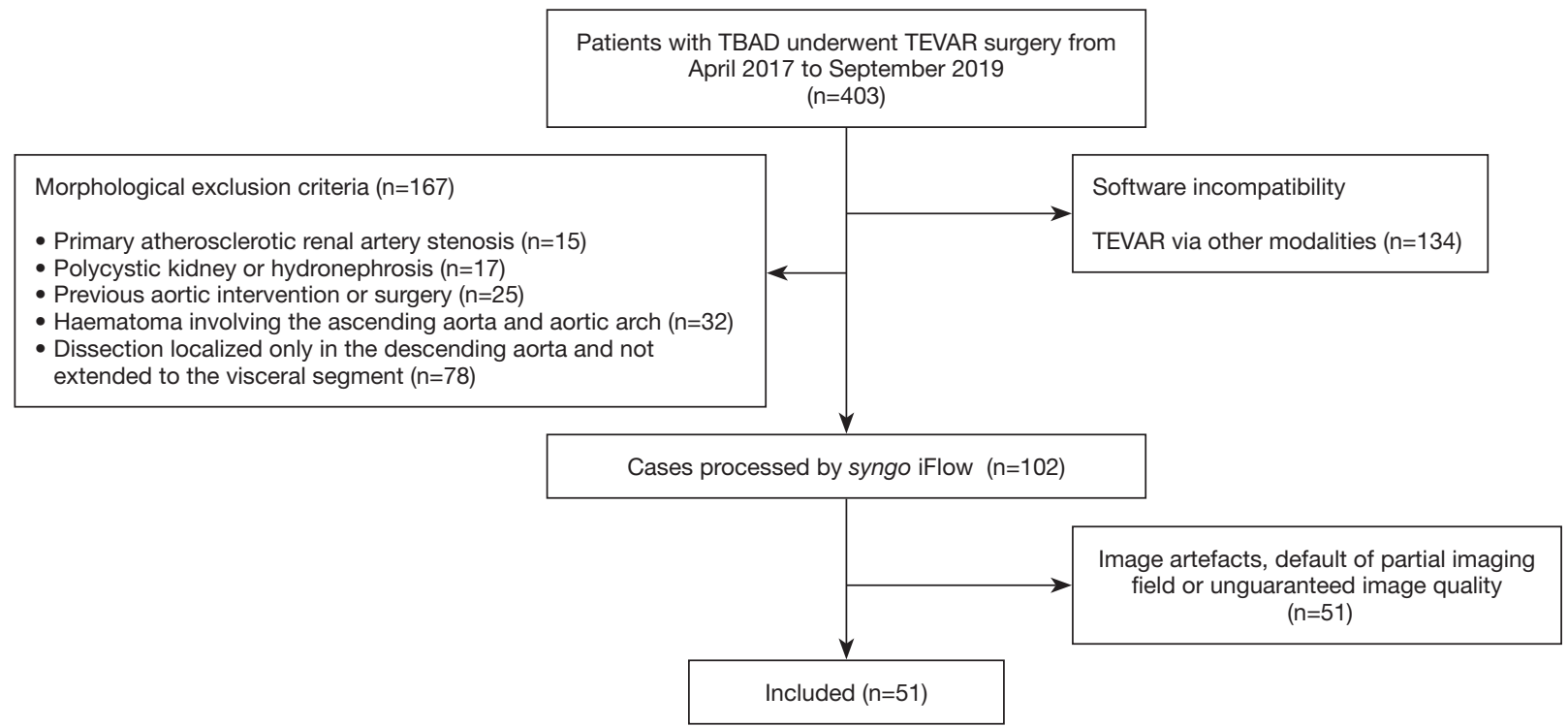

Figure 1 Selection criteria for the cases included in this study. TBAD, type B aortic dissection; TEVAR, thoracic endovascular aortic repair.

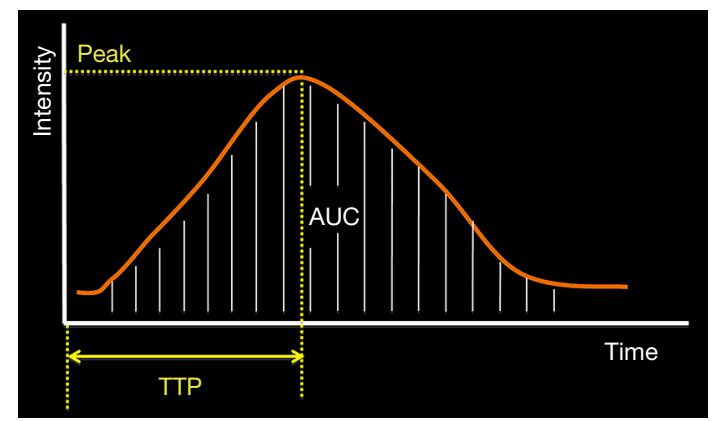

Figure 2 Illustration of the time-intensity curve (TIC) and related parameters, including peak as the maximum intensity value, the time to peak (TTP) contrast intensity, and the area under the curve (AUC).

power injector with a $0.4-\mathrm{s}$ delay. The DSA acquisition duration was controlled manually, typically $12-15 \mathrm{~s}$, to ensure contrast visualization appeared in the bilateral renal cortex. All the above acquisition and injection parameters were kept constant before and after stent-graft implantation for each patient. Also, careful and appropriate control of the respiratory rate, blood pressure, and heart rate were maintained during the entire procedure.

\section{Image post-processing}

Pre- and post-procedural DSA data were transferred to a dedicated workstation (syngo X-WorkplaceVB21; Siemens Healthineers) for quantitative DSA analysis using the syngo iFlow software. syngo iFlow converts a DSA series into one color-coded map, reflecting the progress of contrast intensity changes over time. The delay time from injection of contrast agent to maximum opacification is defined on a pixel-by-pixel basis and then coded as a specific color, ranging from red (early maximum intensity) to blue (late maximum intensity). Once the region of interest (ROI) is selected, the time-intensity curve (TIC) of the ROI (Figure 2) is obtained with a series of representative parameters as follows:

(I) ROI area: area of the ROI $\left(\mathrm{mm}^{2}\right)$;

(II) Ref ROI: reference ROI, which is the first defined ROI assigned by default;

(III) ROI peak/Ref peak: ratio of the maximum intensity value of each specified ROI to the Ref ROI (abbreviated as Pr);

(IV) ROI peak time (also known as the time to peak, TTP), the time when the contrast intensity of the ROI reached maximum value;

(V) dTTP: the TTP of Ref ROI is subtracted from that of each ROI, which was not displayed in the iFlow table, and then calculated manually; and

(VI) ROI AUC/Ref AUC: ratio of the area under the curve (AUC) of every ROI to Ref ROI (abbreviated as AUCr).

In one syngo iFlow color-coded map, nine normalized 


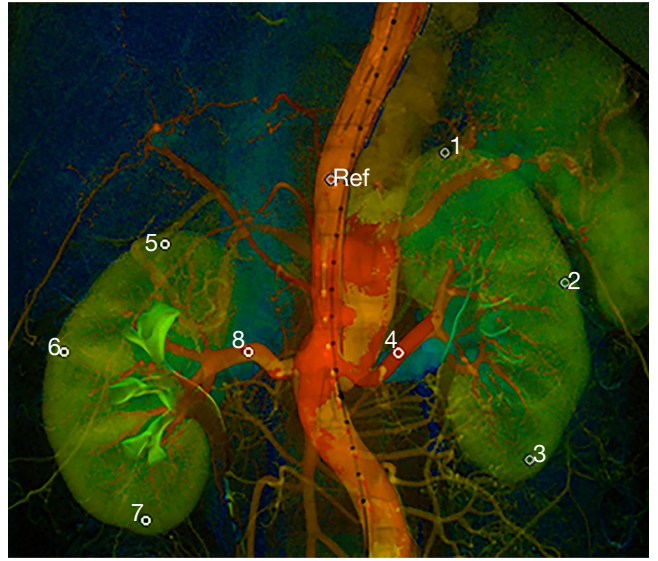

Figure 3 Display of the specific regions of interest (ROIs) in the syngo iFlow color-coded map. Nine ROIs were selected, including the reference (Ref) ROI located in the true lumen of the abdominal aorta near T12, ROI [1] and ROI [5] located in the upper pole of the bilateral renal cortex, ROI [2] and ROI [6] located in the midpole of the bilateral renal cortex, ROI [3] and ROI [7] located in the lower pole of the bilateral renal cortex, and ROI [4] and ROI [8] located in the bilateral renal arteries.

ROIs were defined as follows (Figure 3):

(I) Ref ROI: located in the true lumen of the abdominal aorta near T12 and kept constant for each measurement;

(II) ROI [1] and ROI [5]: located in the upper pole of the bilateral renal cortex;

(III) ROI [2] and ROI [6]: located in the mid-pole of the bilateral renal cortex;

(IV) ROI [3] and ROI [7]: located in the lower pole of the bilateral renal cortex; and

(V) ROI [4] and ROI [8]: located in the bilateral renal arteries.

For a more accurate evaluation of renal perfusion, ROIs were drawn in the kidney cortex area that was regarded as parenchyma with little interference from overlapping vessels. If an accessory renal artery existed, an extra ROI would be drawn within the artery.

\section{Data and statistical analysis}

For the six ROIs in the bilateral kidney cortex, the mean values of the parameters were calculated as the representative indicators of the renal cortex, including the average delayed time to peak (avg.dTTP), average peak ratio (avg.Pr), and average area under the curve ratio (avg.
AUCr). Likewise, identical parameters of the other two ROIs in the bilateral renal artery (or more than 2 ROIs if the accessory renal artery existed) were also calculated and analyzed. Serum creatinine $(\mathrm{Cr})$ and blood urea nitrogen (BUN) before and after TEVAR were collected, and the estimated glomerular filtration rate (eGFR) was calculated after that using the Cockcroft-Gault (CG) formula (7).

Data with a normal distribution were expressed as mean \pm standard deviation, while data with a non-normal distribution were expressed as median and interquartile range. The avg.dTTP, avg.Pr, and avg.AUCr of the renal artery and cortex in the pre-operative syngo iFlow map was compared to the post-operative syngo iFlow map's corresponding values using the Wilcoxon signed-rank test. The significance level for all tests was defined as alpha $=0.05$.

Spearman correlation analysis was used to explore the relationship between BUN or eGFR with each iFlow parameter (including pre-and-post-TEVAR values) with $\mathrm{P}<0.05$ and $95 \%$ confidence intervals considered statistically significant.

All graphs and statistical analyses were derived using GraphPad Prism 7.0 (GraphPad Software, San Diego, CA, USA) and SPSS 25.0 (IBM Corporation, Somers, NY, USA).

\section{Results}

The demographic and clinical baseline data for the included patients are described in Table 1. There were no in-hospital deaths, re-interventions, or paraplegia during the perioperative period for the 51 patients included. One patient had a stroke 5 days after TEVAR. Among the 15 patients with combined acute kidney injury (AKI) before TEVAR, the renal function of 9 patients returned to normal levels without dialysis at discharge, and the other 6 patients required temporary dialysis and fully recovered at the 6-month follow-up. The 1-month postoperative CT scans revealed type I endoleaks in 3 patients (5.9\%), type II endoleaks in 2 patients (3.9\%), and type IV endoleaks in 3 patients $(5.9 \%)$.

An average of $1.35 \pm 0.55$ stent-grafts was implanted in each patient ( 1 stent graft in 39 patients and 2 in 12 patients), with a mean coverage length of $194.69 \pm 33.25$ $\mathrm{mm}$. The median cumulative dose-area product (DAP) during TEVAR was $26,664.95 \mu \mathrm{Gy} \cdot \mathrm{m}^{2}$ (range, 13,355$\left.46,996 \mu \mathrm{Gy} \cdot \mathrm{m}^{2}\right)$. In total, 102 DSA series, including 51 pre-operative and 51 post-operative imaging data, were 
Table 1 Clinical characteristics of patients $(n=51)$

\begin{tabular}{|c|c|}
\hline Variables & Values \\
\hline Gender (males), n (\%) & $43(84.3)$ \\
\hline Age (years), mean \pm SD & $53.35 \pm 12.27$ \\
\hline Timing of TEVAR (days), mean \pm SD & $430.45 \pm 1,351.67$ \\
\hline$<14, \mathrm{n}(\%)$ & $27(52.9)$ \\
\hline $14-60, \mathrm{n}(\%)$ & $13(25.5)$ \\
\hline$>60, \mathrm{n}(\%)$ & $11(21.6)$ \\
\hline BMI $\left(\mathrm{kg} / \mathrm{m}^{2}\right)$, mean $\pm \mathrm{SD}$ & $27.17 \pm 3.68$ \\
\hline Obesity >28, n (\%) & $22(43.1)$ \\
\hline \multicolumn{2}{|l|}{ Comorbidities, n (\%) } \\
\hline Hypertension & $50(98.0)$ \\
\hline COPD & $1(2.0)$ \\
\hline Diabetes mellitus & $8(15.7)$ \\
\hline Coronary heart disease & $19(37.3)$ \\
\hline Acute kidney injury & $15(29.4)$ \\
\hline Accessory renal artery, $\mathrm{n}(\%)$ & $6(11.8)$ \\
\hline \multicolumn{2}{|c|}{ Location of the distal end of dissection, $n(\%)$} \\
\hline Middle descending aorta & $0(0)$ \\
\hline Distal descending aorta & $7(13.7)$ \\
\hline Celiac trunk & $3(5.9)$ \\
\hline Superior mesenteric artery & $2(3.9)$ \\
\hline Renal artery & $10(19.6)$ \\
\hline Iliac artery & $29(56.9)$ \\
\hline \multicolumn{2}{|c|}{ Patterns of involvement of the renal artery (left/right), n (\%) } \\
\hline True lumen & $34(66.7) / 36(70.6)$ \\
\hline False lumen & $7(13.7) / 7(13.7)$ \\
\hline Both true and false lumens & $10(19.6) / 8(15.7)$ \\
\hline \multicolumn{2}{|l|}{ In-hospital outcomes, n (\%) } \\
\hline Death & $0(0)$ \\
\hline Stroke & $1(2.0)$ \\
\hline Paraplegia & $0(0)$ \\
\hline Acute renal failure & $6(11.8)$ \\
\hline
\end{tabular}

SD, standard deviation; TEVAR, thoracic endovascular aortic repair; BMI, body mass index; COPD, chronic obstructive pulmonary disease. successfully post-processed. The pre-and post-operative avg.dTTPs, avg.Prs, and avg.AUCrs of the renal artery and cortex are shown in Figure 4. After TEVAR, syngo iFlow showed a significant $33.0 \%$ increase in avg. $\operatorname{Pr}(\mathrm{P}<0.001)$ and a significant $35.1 \%$ increase in avg. $\mathrm{AUCr}(\mathrm{P}<0.001)$ in the renal artery. Additionally, there was a significant $12.2 \%$ decrease in the avg.dTTP $(\mathrm{P}=0.001)$, a significant $24.5 \%$ increase in avg. $\operatorname{Pr}(\mathrm{P}=0.004)$, and a significant $38.3 \%$ increase in avg.AUCr $(\mathrm{P}=0.009)$ in the renal cortex. Typical images of pre-and post-operative syngo iFlow color-coded maps are shown in Figure 5. No statistically significant correlation was found between pre-operative iFlow parameters and renal function variables. Nevertheless, after TEVAR, there was a statistical correlation between the avg. Pr of the renal artery and eGFR $(r=0.30 ; P=0.0349)$, the avg.Pr of the renal cortex and eGFR $(r=0.30 ; P=0.0300)$, and the avg.AUCr of the renal cortex and BUN ( $\mathrm{r}=0.31$; $\mathrm{P}=0.0289$; Tables 2,3).

\section{Discussion}

Renal malperfusion is a crucial complication of TBAD, which is caused by static or dynamic occlusion of branched vessels, leading to reduced blood flow to the tissues (8). Generally, multiple imaging modalities, including CT, MRI, ultrasound, and DSA, can be used to evaluate renal blood flow and perfusion (9). However, CT or MRI cannot be performed during operations. Moreover, excessive contrast media and $\mathrm{X}$-ray doses are unavoidable during CT perfusion imaging. Although ultrasound is feasible to measure blood flow speed and initially assess renal perfusion, it is not easy to achieve real-time and quantitative evaluations $(9,10)$. In this study, syngo iFlow provided an effective means for surgeons to identify the flow changes in the visceral arteries and the abdominal aorta in real-time during the operation. Using syngo iFlow to evaluate hemodynamics and renal perfusion resulted in several benefits. First, syngo iFlow provided real-time analysis. The color-coded images would be displayed as soon as the DSA series was acquired, and all measurements could be calculated immediately once the ROIs were selected. In some instances, the hemodynamic changes could not be thoroughly determined using intuitive observations of the color-coded maps, and in such cases, the quantitative parameters were useful complements. 
A Avg.dTTP of renal artery
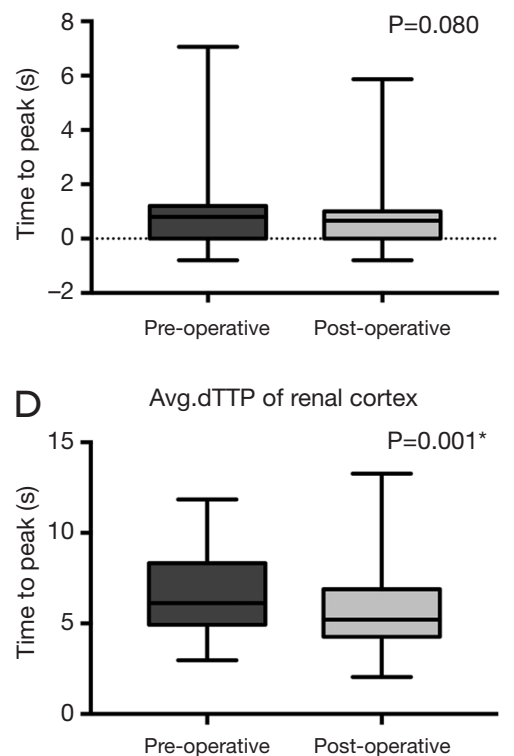

B

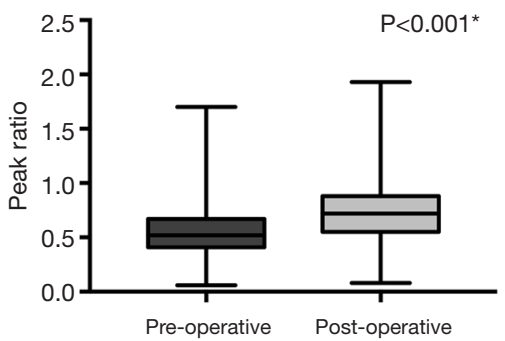

$\mathrm{E}$

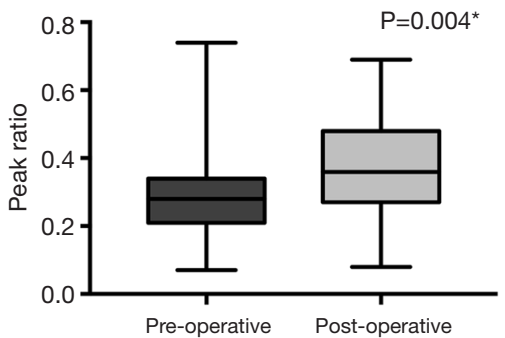

C Avg.AUCr of renal artery

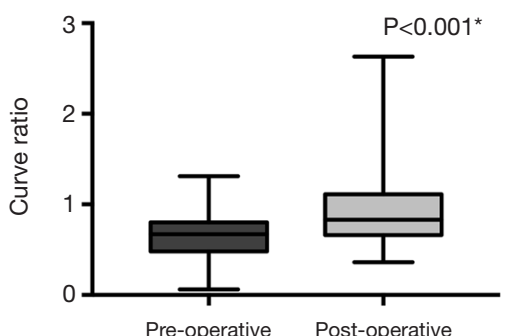

F Avg.AUCr of renal cortex

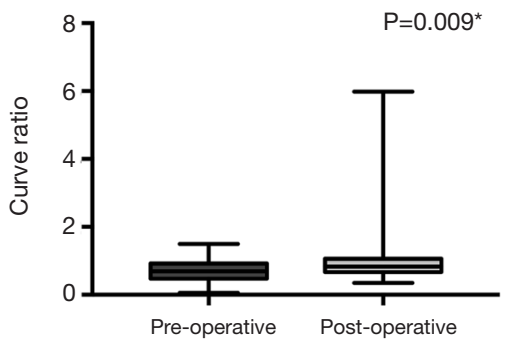

Figure 4 Wilcoxon signed rank test of pre- and post-operative measurements using syngo iFlow. (A) The difference between the pre- and post-operative avg.dTTP of the renal artery $(1.02 \pm 1.56$ versus $0.72 \pm 1.05 \mathrm{~s}, \mathrm{P}=0.080)$; (B) the difference between the pre- and post-operative avg.Pr of the renal artery $(0.56 \pm 0.28$ versus $0.75 \pm 0.32, \mathrm{P}<0.001)$; $(\mathrm{C})$ the difference between the pre- and post-operative avg.AUCr of the renal artery $(0.66 \pm 0.26$ versus $0.90 \pm 0.38, \mathrm{P}<0.001)$; (D) the difference between the pre- and post-operative avg.dTTP of the renal cortex $(6.52 \pm 2.09$ versus $5.73 \pm 2.04 \mathrm{~s}, \mathrm{P}=0.001)$; (E) the difference between the pre- and post-operative avg.Pr of the renal cortex $(0.30 \pm 0.13$ versus $0.37 \pm 0.14, \mathrm{P}=0.004)$; $(\mathrm{F})$ the difference between the pre- and post-operative avg.AUCr of the renal cortex $(0.72 \pm 0.32$ versus $0.99 \pm 0.80$, $\mathrm{P}=0.009)$. *, $\mathrm{P}<0.05$. avg.dTTP, average delayed time to peak; avg.Pr, average peak ratio; avg.AUCr, average area under the curve ratio.
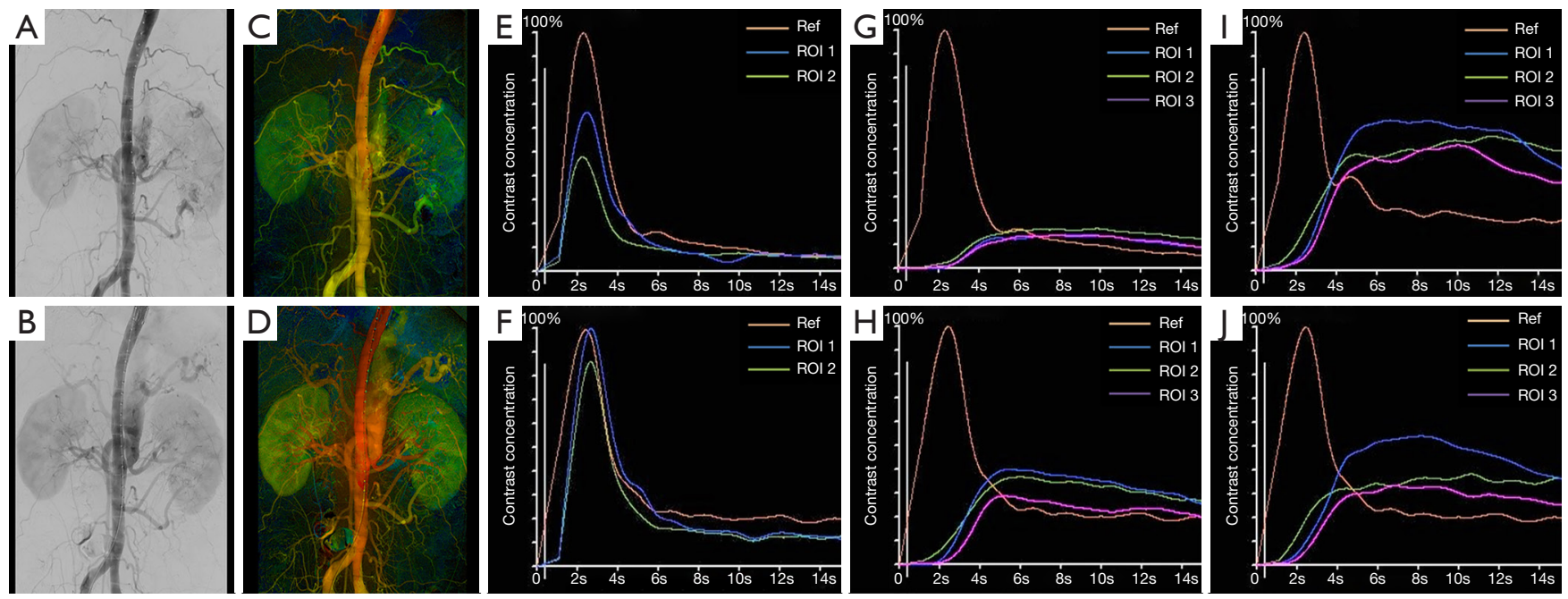

Figure 5 Imaging analysis of a typical patient undergoing endovascular treatment for TBAD. A comparison of the pre- and post-operative original DSA images $(\mathrm{A}, \mathrm{B})$ and the syngo iFlow color-coded maps (C,D). A comparison of the pre- and post-operative time-intensity curves of the renal arteries $(\mathrm{E}, \mathrm{F})$, the right renal cortex $(\mathrm{G}, \mathrm{H})$, and the left renal cortex $(\mathrm{I}, \mathrm{J})$. In these curves, the $\mathrm{x}$-axis refers to time (second) and $y$-axis represents the ratio of intensity within the ROI. ROI 1 and ROI 2 (E,F) were located in the renal arteries; ROI 1, ROI 2, and ROI 3 $(\mathrm{G}, \mathrm{H}, \mathrm{I}, \mathrm{J})$ were located in the renal cortex. TBAD, type B aortic dissection; DSA, digital subtraction angiography; ROI, region of interest. 
Table 2 Spearman correlation analyses of eGFR with average dTTP, average Pr, and average AUCr

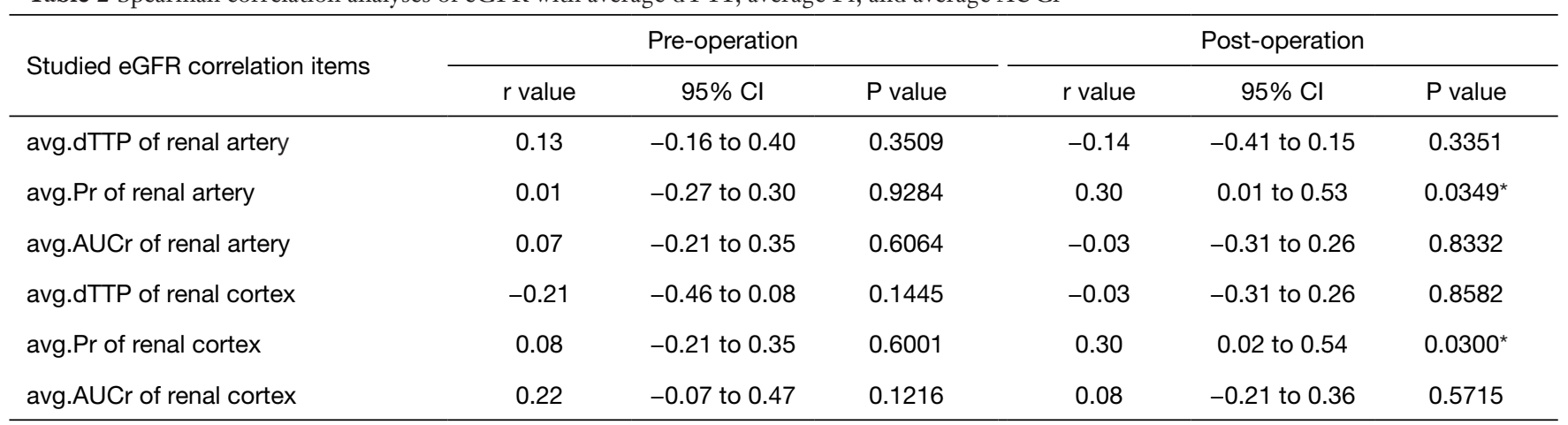

*, $\mathrm{P}<0.05$. eGFR, estimated glomerular filtration rate (Cockcroft-Gault formula); Cl, confidence interval; avg.dTTP, average delayed time to peak; avg.Pr, average peak ratio; avg.AUCr, average area under the curve ratio.

Table 3 Spearman correlation analyses of BUN with average dTTP, average Pr and average AUCr

\begin{tabular}{|c|c|c|c|c|c|c|}
\hline Studied BUN correlation items & \multicolumn{3}{|c|}{ Pre-operation } & \multicolumn{3}{|c|}{ Post-operation } \\
\hline avg.dTTP of renal artery & -0.09 & -0.36 to 0.20 & 0.5280 & -0.00 & -0.29 to 0.28 & 0.9804 \\
\hline avg.Pr of renal artery & 0.18 & -0.10 to 0.44 & 0.1953 & -0.03 & -0.31 to 0.25 & 0.8130 \\
\hline avg.AUCr of renal artery & 0.11 & -0.18 to 0.38 & 0.4318 & 0.14 & -0.15 to 0.41 & 0.3345 \\
\hline avg.Pr of renal cortex & 0.08 & -0.21 to 0.36 & 0.5652 & 0.21 & -0.08 to 0.47 & 0.1369 \\
\hline avg.AUCr of renal cortex & -0.05 & -0.33 to 0.24 & 0.7280 & 0.31 & 0.03 to 0.54 & $0.0289^{*}$ \\
\hline
\end{tabular}

*, $\mathrm{P}<0.05$. BUN, blood urea nitrogen; $\mathrm{Cl}$, confidence interval; avg.dTTP, average delayed time to peak; avg.Pr, average peak ratio; avg. AUCr, average area under the curve ratio.

Second, syngo iFlow allowed surgeons to evaluate blood flow patency and measure quantitative dynamic changes in renal parenchyma intra-operatively, which facilitated efficient evaluation if additional procedures were required. Third, no extra dose of contrast media was required in this application, which reduced kidney injury risk, especially for patients with chronic kidney disease.

According to the Wilcoxon signed-rank test results, both the avg.AUCr and avg.Pr of the renal artery increased significantly after the stent-graft placement, but the avg. dTTP was unchanged. One possible reason is that the ROI in the renal artery was so close to the Ref ROI in the aorta that any changes in avg.dTTP was minimal when the blood flow varied. It is interesting to note that the TIC of the ROI within the lumen showed typical wash-in and wash-out performance with a remarkable peak value.

On the contrary, the TIC of the ROI within the renal cortex showed visible wash-in tendency in the early phase but poor wash-out shape, which diminished the validity of the peak value definition and the TTP measurement (Figure 5G,H,I,f). According to physiology literature, perfusion is defined as the volume of blood passing through an organ at a certain time (11). In contrast to flow parameters for transient analysis, the AUC is the integral measure of the signal intensity against the acquisition time and is proportional to the volume of blood passing through a region $(12,13)$. Therefore, the avg.AUCr is more appropriate for demonstrating renal perfusion changes. However, since the AUC, as an estimation of volume flow, can also be affected by the diameter of the feeding artery and the kidney's resistance, it may be beneficial to investigate the clinical value of the AUC further.

Spearman correlation analyses revealed no statistically significant correlation between pre-operative iFlow parameters and renal function variables. In contrast, positive relationships were shown between post-operative 
iFlow parameters and renal function variables (avg.Pr of the renal artery and eGFR, avg.Pr of the renal cortex and eGFR, avg. AUCr of the renal cortex and BUN), suggesting that iFlow measurements in real-time may be useful in the evaluation and prediction of kidney outcomes after TEVAR. However, the results indicated relatively low correlation coefficients, which may be impacted by sample size. Moreover, perioperative serologic indexes may not accurately reflect renal function's tendency, especially in the early phase of AKI. Therefore, long-term follow-up data and renal function surveillance are essential further to verify the predictive value of iFlow in renal perfusion.

In this study, several conditions need to be carefully controlled to develop a standardized procedure. First, the injection protocol, including the injection rate, injection pressure, catheter position, and amount of contrast media, had to be consistent. Second, the patient's blood pressure must be controlled during DSA acquisition before and after operations because any change in blood pressure directly affects blood flow. Third, motion and bowel gas artifacts should be reduced, as these artifacts could lead to blurred syngo iFlow images, making a precise selection of ROIs within a specific region difficult. Some parameters need to be calculated from basic syngo iFlow data, and real-time automatic data processing would have been beneficial.

In future studies, larger samples and long-term followup data are necessary, and subgroup analyses should be performed to explore further the relationship between the pathological conditions and the renal hemodynamics changes.

\section{Conclusions}

In summary, this study demonstrated that syngo iFlow provided a novel quantitative method for evaluating renal hemodynamic changes in patients with TBAD undergoing endovascular treatment. Furthermore, TIC parameters may facilitate the intraprocedural evaluation of renal blood flow and perfusion to complement the color-coded maps.

\section{Acknowledgments}

The authors would like to thank Dr. Dongting Ye and Dr. Zhiming Feng for recording the medical data, and Dr. Qi Sun and Mr. Wanmu Liu for their work in processing the angiography data.

Funding: This work was supported by grants from the Chinese Academy of Medical Sciences (CAMS) Initiative for Innovative Medicine, China (NO. 2016-I2M-1-016) and the National Natural Science Foundation of China (81870345).

\section{Footnote}

Conflicts of Interest: All authors have completed the ICMJE uniform disclosure form (available at http://dx.doi. org/10.21037/qims-20-992). The authors have no conflicts of interest to declare.

Ethical Statement: The authors are accountable for all aspects of the work in ensuring that questions related to the accuracy or integrity of any part of the work are appropriately investigated and resolved. This study was conducted following the Declaration of Helsinki (as revised in 2013). The retrospective study was approved by the National Center for Cardiovascular Diseases of China, the Ethics Committee (NO. 2018-1119), and the institutional review board. Patient informed consent was waived due to the retrospective nature of the study.

Open Access Statement: This is an Open Access article distributed in accordance with the Creative Commons Attribution-NonCommercial-NoDerivs 4.0 International License (CC BY-NC-ND 4.0), which permits the noncommercial replication and distribution of the article with the strict proviso that no changes or edits are made and the original work is properly cited (including links to both the formal publication through the relevant DOI and the license). See: https://creativecommons.org/licenses/by-nc-nd/4.0/.

\section{References}

1. Jonker FH, Patel HJ, Upchurch GR, Williams DM, Montgomery DG, Gleason TG, Braverman AC, Sechtem U, Fattori R, Di Eusanio M, Evangelista A, Nienaber CA, Isselbacher EM, Eagle KA, Trimarchi S. Acute type $\mathrm{B}$ aortic dissection complicated by visceral ischemia. J Thorac Cardiovasc Surg 2015;149:1081-6.e1.

2. Dake MD, Kato N, Mitchell RS, Semba CP, Razavi MK, Shimono T, Hirano T, Takeda K, Yada I, Miller DC. Endovascular stent-graft placement for the treatment of acute aortic dissection. N Engl J Med 1999;340:1546-52.

3. Shu C, Fang K, Luo M, Li Q, Wang Z. Emergency endovascular stent-grafting for acute type $\mathrm{B}$ aortic dissection with symptomatic malperfusion. Int Angiol 2013;32:483-91. 
4. Zhang XB, Zhuang ZG, Ye H, Beilner J, Kowarschik M, Chen JJ, Chi JC, Xu JR. Objective assessment of transcatheter arterial chemoembolization angiographic endpoints: preliminary study of quantitative digital subtraction angiography. J Vasc Interv Radiol 2013;24:667-71.

5. Zhou M, Su Z, Shi Z, Fu W, Meng X, Wang Y, Guo B, Huang K. Application of color-coded quantitative digital subtraction angiography in predicting the outcomes of immediate type I and type III endoleaks. J Vasc Surg 2017;66:760-7.

6. Burkhardt JK, Chen X, Winkler EA, Weiss M, Yue JK, Cooke DL, Kim H, Lawton MT. Early Hemodynamic Changes Based on Initial Color-Coding Angiography as a Predictor for Developing Subsequent Symptomatic Vasospasm After Aneurysmal Subarachnoid Hemorrhage. World Neurosurg 2018;109:e363-73.

7. Cockcroft DW, Gault MH. Prediction of creatinine clearance from serum creatinine. Nephron 1976;16:31-41.

8. Kamman AV, Yang B, Kim KM, Williams DM, Michael Deeb G, Patel HJ. Visceral Malperfusion in Aortic

Cite this article as: Fang K, Zhao J, Luo M, Xue Y, Wang H, Ye L, Zhang X, Zheng L, Shu C. Quantitative analysis of renal blood flow during thoracic endovascular aortic repair in type B aortic dissection using syngo iFlow. Quant Imaging Med Surg 2021;11(8):3726-3734. doi: 10.21037/qims-20-992
Dissection: The Michigan Experience. Semin Thorac Cardiovasc Surg 2017;29:173-8.

9. Grenier N, Cornelis F, Le Bras Y, Rigou G, Boutault JR, Bouzgarrou M. Perfusion imaging in renal diseases. Diagn Interv Imaging 2013;94:1313-22.

10. Thomas S, Jakob S. Can we measure renal tissue perfusion by ultrasound? J Med Ultrasound 2009;17:9-16.

11. Scholbach T, Dimos I, Scholbach J. A new method of color Doppler perfusion measurement via dynamic sonographic signal quantification in renal parenchyma. Nephron Physiol 2004;96:p99-104.

12. Zhuang H, Yang ZG, Wang ZQ, Wang XD, Chen HJ, Zhang YC, Luo Y. Features of time-intensity curve parameters of colorectal adenocarcinomas evaluated by double-contrast enhanced ultrasonography: initial observation. Eur J Radiol 2012;81:677-82.

13. Kim AH, Shevitz AJ, Morrow KL, Kendrick DE, Harth $\mathrm{K}$, Baele H, Kashyap VS. Characterizing tissue perfusion after lower extremity intervention using two-dimensional color-coded digital subtraction angiography. J Vasc Surg 2017;66:1464-72. 